(1)

CrossMark

\title{
Management of pulmonary toxicity associated with immune checkpoint inhibitors
}

\author{
Myriam Delaunay ${ }^{1}$, Grégoire Prévot ${ }^{1}$, Samia Collot $^{2}$, Laurent Guilleminault ${ }^{1}$, \\ Alain Didier ${ }^{1}$ and Julien Mazières ${ }^{1}$
}

Affiliations: ${ }^{1}$ Respiratory Disease Dept, Larrey Hospital, University Hospital of Toulouse, Paul Sabatier University, Toulouse, France. ${ }^{2}$ Radiology Dept, Rangueil Hospital, University Hospital of Toulouse, Toulouse, France.

Correspondence: Julien Mazières, Thoracic Oncology Unit, Respiratory Disease Dept, Hôpital Larrey, CHU Toulouse, Chemin de Pouvourville, 31059 Toulouse, France. E-mail: mazieres.jachu-toulouse.fr

@ERSpublications

Immune-related lung toxicity can be life-threatening. Early recognition and management including CT scan and bronchoscopy should be encouraged. Treatment mainly relies on steroids. In addition, re-challenge should be discussed in multidisciplinary boards. http://bit.ly/2WNYH8I

Cite this article as: Delaunay M, Prévot G, Collot S, et al. Management of pulmonary toxicity associated with immune checkpoint inhibitors. Eur Respir Rev 2019; 28: 190012 [https://doi.org/10.1183/ 16000617.0012-2019]

ABSTRACT Immunotherapy has become a standard of care in oncology, following the recent approvals of cytotoxic T-lymphocyte-associated protein-4 and programmed cell death-1 inhibitors in lung cancer, melanoma, renal cell carcinoma, Hodgkin's lymphoma, bladder, head and neck cancers. Besides their efficacy, these agents also generate specific immune-related adverse events. Due to the increasing prescription of immune-checkpoint inhibitors, the incidence of immune toxicity will continue to rise. The awareness of immune-related adverse events is key to ensuring both diagnosis and management of the possible serious adverse events. Although severe immune-related adverse events remain rare, they can lead to discontinued treatment or to death if they are not forecasted and managed properly. Even if lung toxicity is not the most frequent adverse event, it remains critical as it can be life-threatening. Herein, the main aspects of pulmonary toxicity are reviewed and guidelines are also proposed in order to manage the possible side-effects.

\section{Introduction}

Anti-tumour immunotherapy and especially immune-checkpoint inhibitors (ICIs) now take part in the therapeutic strategy of nonsmall cell lung cancer (NSCLC) and other advanced solid tumours [1-3]. Therefore, due to the increasing use of ICIs, many oncologists may face side-effects.

ICIs present a favourable overall benefit-to-risk profile; however, they are linked to specific toxicities in terms of mechanisms of action, known as immune-related adverse events, greatly differing from the toxicities observed with conventional cytotoxic chemotherapies or targeted therapies. An immune-related adverse event may potentially affect any tissue but the skin, endocrine system, gastrointestinal tract or lung are the most commonly involved [4]. To date, no guidelines have been specifically provided for lung toxicity. According to the literature and considering our work and expertise as pulmonologists, radiologists and oncologists our objective is to highlight lung toxicity and share our experience on its management.

Provenance: Submitted article, peer reviewed.

Received: 12 Feb 2019 | Accepted after revision: 08 June 2019

Copyright (CERS 2019. This article is open access and distributed under the terms of the Creative Commons Attribution Non-Commercial Licence 4.0. 
What is the incidence of pulmonary toxicity associated with ICI treatment?

Cases of interstitial lung disease (ILD) induced by ICIs (ICI-ILD) have been reported since the first clinical trials on both anti-programmed cell death (PD)-1 and anti-programmed death-ligand (PD-L)1 antibodies. According to a meta-analysis, the overall ICI-ILD incidence was $2.7 \%$ for all grades and $0.8 \%$ for the most severe grades (grade $\geqslant 3$ ). The overall incidence of ICI-ILD for all grades was between $1.4 \%$ and 5.8\% in NSCLC studies, whereas in melanoma studies it was between $1 \%$ and $4.4 \%$ and in renal cell carcinoma studies it was between $2.7 \%$ and $4.8 \%$ [5]. According to our daily practice, three recent series have shown higher ILD incidences $(3.5 \%, 5 \%$ and $11.8 \%$, respectively) [6-8]. It is well known that the incidence increased in patients treated with combination immunotherapy as observed in melanoma trials. The incidence of pneumonitis was higher in combination regimens (including nivolumab and ipilimumab, given concurrently or sequentially, or nivolumab plus peptide vaccines) than in monotherapy (6.6\%) [5]. However, a combination of immunotherapy (pembrolizumab) with chemotherapy was not linked to an increased risk of pneumonitis in lung cancer [9]. Moreover, the administration of immunotherapy (durvalumab) after chemoradiation in patients with locally advanced NSCLC was related to an acceptable rate of lung toxicity (3.4\% grade $3 / 4$ versus $2.6 \%$ in the placebo arm) $[10,11]$.

\section{Can we forecast or predict pulmonary toxicity?}

Before prescribing ICIs, it is important to identify the potential risk factors that may favour ICI-ILD.

ILD appeared to be more frequent in patients with NSCLC than in patients with melanoma (4.1\% versus $1.6 \%, \mathrm{p}=0.002$ ), with an earlier time to onset [8]. Tobacco smoke exposure or chronic lung diseases in patients with lung cancer could explain this difference. Lung tumour burden may also limit pulmonary endurance to external stress factors and promote pulmonary toxicity [11].

The following study assessed nivolumab safety in patients with NSCLC and idiopathic interstitial pneumonia. It included six patients and none of them developed any grade of pneumonitis; however, this result should be confirmed in a larger cohort [12]. Other parameters, such as COPD or a radiotherapy history, which are often associated with a higher risk of pneumonitis in patients receiving epidermal growth factor receptor tyrosine-kinase inhibitors, have not been identified to date for ICIs. Thoracic radiotherapy may increase local inflammation and release tumour antigens into pulmonary tissues and thus potentiate pulmonary toxicity [13]. It is important to monitor respiratory functions before starting immunotherapy in patients with COPD. Another meta-analysis suggests a higher incidence of any grade of toxicity and greater severity of ILD with anti-PD-1 treatment compared with anti-PD-L1 [14]. The sparing of PD-1/PD-L2 interactions with PD-L1 inhibitors may explain this finding [15]. The previous meta-analysis also highlights a significantly higher rate of all-grade pneumonitis in treatment-naive patients [14]. This area needs further investigation in the light of recent US Food and Drug Administration (FDA) approval of first-line chemotherapy (either as single agent or in combination with chemotherapy). As the incidence is also higher with combined treatments rather than monotherapy, patients treated with bi-therapy (anti-PD-1 and anti-cytotoxic T-lymphocyte-associated protein (CTLA)-4) need close clinical and radiological follow-up [5].

Dysimmune diseases are usually contraindications for immunotherapy, except for type 1 diabetes, for hypothyroidism requiring treatment only with hormonal substitution and skin diseases requiring only topical treatment (e.g. vitiligo and psoriasis). JoHnson et al. [16] evaluated immune-related adverse event frequency in a retrospective review of patients treated for advanced melanoma with ipilimumab and with pre-existing autoimmune disorders. They included 30 patients with autoimmune disorders such as systemic lupus erythematosus, multiple sclerosis, rheumatoid arthritis, inflammatory bowel disease, psoriasis and autoimmune thyroiditis. A total of $50 \%$ of patients presented with neither autoimmune disease flares nor immune-related adverse events. Overall, $27 \%$ of patients needed steroid treatment due to autoimmune disease exacerbations. $33 \%$ of patients presented with grade 3 to 5 immune-related adverse events (especially colitis, hypophysitis and thyroiditis) being reversible with corticotherapy and, in two cases, with infliximab [16]. MeNzIEs et al. [17] included 52 patients with advanced melanoma treated with anti-PD-1 and pre-existing autoimmune disorders (such as rheumatoid arthritis, Sjogren's syndrome, immune thrombocytopaenic purpura and psoriasis). Furthermore, 38\% showed other flare autoimmune disorders and $29 \%$ had other immune-related adverse events. Only six patients needed to discontinue the treatment (two due to flares and four due to new immune-related adverse events) and there were no treatment-related deaths [17]. More recently, LeONARDi et al. [18] identified 56 patients with NSCLC treated with anti-PD-L1 with an autoimmune disease. An autoimmune disease exacerbation occurred in $13(23 \%)$ patients and four of them needed systemic steroids. A total of $21(38 \%)$ patients developed a mild immune-related adverse event (74\% grade 1-2) and eight patients required steroids [18]. Therefore, immunotherapy may be considered in patients with immune disorders with a very close follow-up; however, the results need to be confirmed in prospective studies. 
Other potential risk factors for dysimmunity should be identified, such as opportunistic pathogenic agents, drugs or occupational exposures. Before initiating ICIs, thorough research of family or personal medical history is required to screen potential autoimmune disorders.

Finally, no biomarker predicting the occurrence of lung toxicity has been identified to date.

\section{How to diagnose ILD associated with ICI treatment? \\ Time to onset and symptomatology}

ICI-ILD symptoms remain unspecific, therefore differential diagnoses are possible. Clinicians should beware of lately appearing respiratory symptoms while treating patients with ICIs. Alerting clinical signs are usually a dry cough, progressive dyspnoea and fine inspiratory crackles. As most cases of ICI-ILD occur during the first 2 months of treatment, clinicians should pay particular attention to the start of treatment.

NisHino et al. [8] described ICI-ILD in 20 patients (10 cases of melanoma, six of lymphoma and four of NSCLC) with a median time to onset of 2.6 months. In a study performed by NAIDOO et al. [19], 43 cases of ICI-ILD were assessed, most of them with presenting melanoma $(n=26)$ or NSCLC $(n=9)$, the median time to onset was 2.8 months. We carried out a study in 24 centres including stage IV NSCLC ( $\mathrm{n}=48$ ), metastatic melanoma $(n=13)$ and three other types of cancer, showing that ICI-ILD occurred in $3.5 \%(64$ out of 1826 cases). The median time to onset was 2.3 months and was significantly earlier in patients with NSCLC (2.1 months) than in patients with melanoma (5.2 months) ( $\mathrm{p}=0.02)$ (table 1) [6].

\section{Radiological patterns}

The main differential diagnoses are tumour progression, pulmonary infections and, in cases of dyspnoea, pulmonary embolism. Thus, we highly recommend performing a high-resolution computed tomography (CT) scan with a contrast agent to rule out differential diagnoses and provide evidence for ICI-ILD. In these cases, CT scan changes mainly include ground-glass opacities and condensations [8]. In the series by NisHiNo et al. [8], radiological analysis found an organising pneumonitis pattern in $65.5 \%$, nonspecific interstitial pneumonitis pattern in $15 \%$, and hypersensitivity pneumonitis pattern in $10 \%$. According to NAIDOO et al. [7], the most frequent patterns were ground-glass opacities in $37 \%$, organising pneumonitis in $19 \%$, and hypersensitivity pneumonitis pattern in $22 \%$.

In our study, we found organising pneumonitis pattern in $23 \%$, hypersensitivity pneumonitis in $16 \%$ and no suggestive pattern in 36\% [6]. Most of the representative radiological patterns are presented in figure 1. Comparisons between these three studies are detailed in both table 1 and figure 2 . The imaging findings follow the classification of interstitial pneumonia according to the American Thoracic Society/European Respiratory Society classification [20]. The organising pneumonitis pattern was defined by peripheral consolidations and air bronchograms as the main CT features. The nonspecific interstitial pneumonitis pattern was defined by several CT scan features, such as ground-glass opacities with marked traction bronchiectasis. Peribronchovascular predominance with subpleural sparing is also described. Furthermore, the hypersensitivity pattern is shown by centrilobular nodules or a bronchiolitis-like appearance $[8,20]$.

\section{TABLE 1 Comparison between the three studies}

\begin{tabular}{|c|c|c|c|}
\hline Study & NAidoo et al. [7] & Nishino et al. [8] & Delaunay et al. [6] \\
\hline Cases n & 43 & 20 & 64 \\
\hline \multirow{7}{*}{ Histology } & 26 Melanoma & 10 Melanoma & 13 Melanoma \\
\hline & 9 NSCLC & 4 NSCLC & 48 NSCLC \\
\hline & $\begin{array}{l}4 \text { Haematological } \\
\text { malignancies }\end{array}$ & 6 Lymphoma & 1 Lymphoma \\
\hline & 1 Bladder carcinoma & & $\begin{array}{l}2 \text { Head and neck } \\
\text { carcinoma }\end{array}$ \\
\hline & 1 Breast carcinoma & & \\
\hline & 1 Pancreatic carcinoma & & \\
\hline & 1 Head and neck carcinoma & & \\
\hline Incidence & $5 \%$ & $11.8 \%$ & $3.5 \%$ \\
\hline $\begin{array}{l}\text { Time to pneumonitis } \\
\text { onset }\end{array}$ & $\begin{array}{l}2.8 \text { months ( } 9 \text { days to } \\
19.2 \text { months) }\end{array}$ & 2.6 months $(0.5-11.5)$ & 2.3 months (0.2-27.4) \\
\hline
\end{tabular}

Data presented as median (range), unless otherwise stated. NSCLC: nonsmall cell lung cancer. 


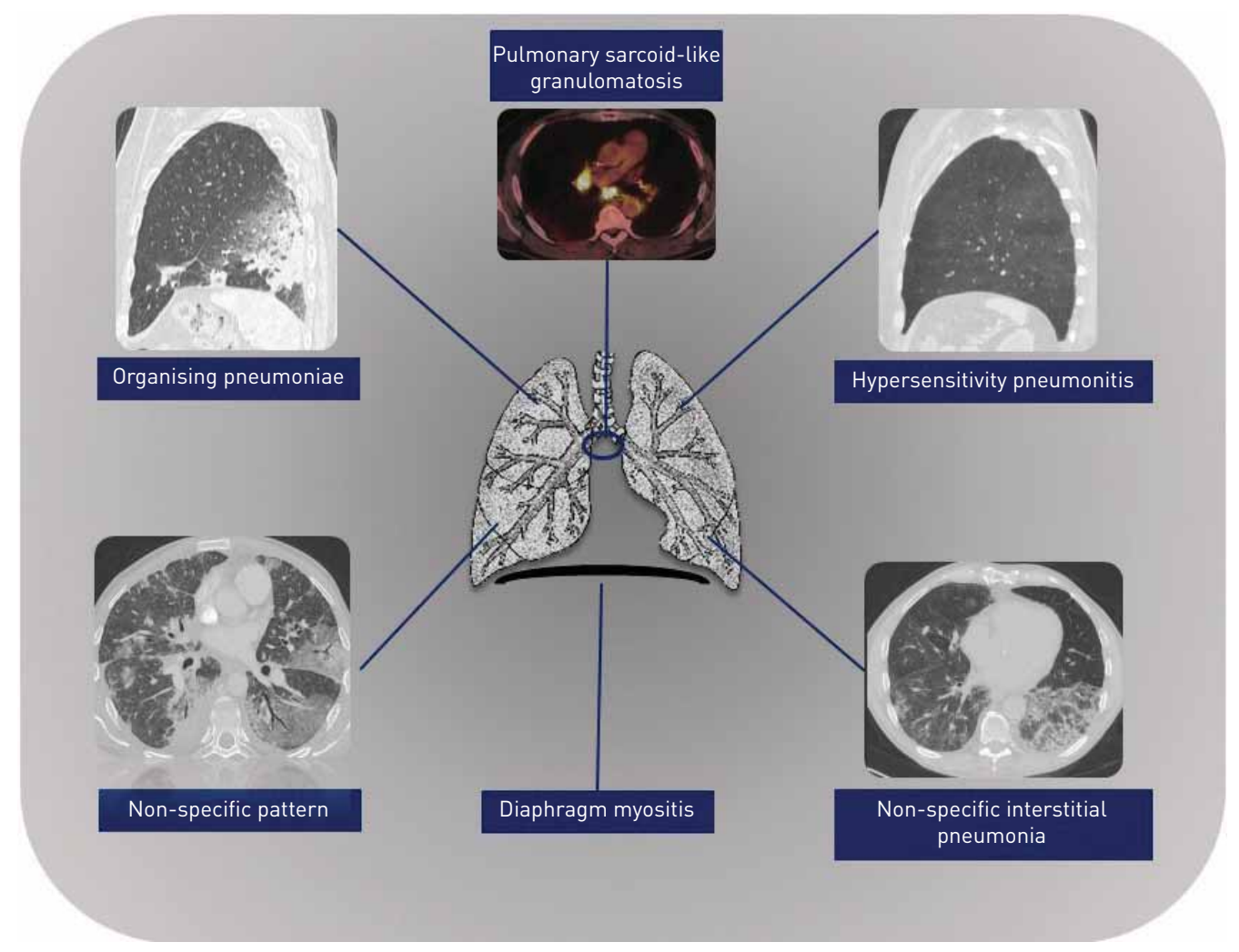

FIGURE 1 Main respiratory toxicities linked to immunotherapy.

\section{Cytopathological criteria}

We recommend the early use of bronchoscopy to obtain a bronchoalveolar lavage. Distal biopsies may also help to rule out differential diagnosis, such as infection or tumour progression, as well as provide evidence regarding ICI-ILD, such as a high percentage of lymphocytes and a T-cell infiltrate (figure 3 ). In our series, we found a predominance of lymphocytes in bronchoalveolar lavages, and biopsies showed lymphocytic infiltration [6]. Some case reports have shown an increase in activated T-cells in the bronchoalveolar lavage of patients with organising pneumonia. This cell population may be involved in this complication pathogenesis [21]. Histopathological findings, when possibly reached, were numerous: cellular interstitial pneumonitis, organising pneumonia, diffuse alveolar damages, and sometimes no specific abnormalities were identified [7, 8, 22].

The high number of lymphocytes contained in bronchoalveolar lavage fluid and the rapid clinical improvement after administration of systemic steroids or treatment withdrawal suggests an immune-mediated mechanism for ILD [23]. The European Society for Medical Oncology (ESMO) recommendations state that a surgical lung biopsy using video-assisted thoracoscopic surgery may be used to secure specific diagnosis [24]. We suggest that transbronchial per-endoscopy biopsies should instead be recommended according to the 2018 American Society of Clinical Oncology (ASCO) clinical practice guidelines [25]. In typical cases, early steroid treatment without invasive procedures may be proposed. Anyway, both biopsy indications and the selection of the most accurate procedures should be discussed within a multidisciplinary board that includes pulmonologists.

\section{How to treat ILD-|C|}

There are no validated recommendations for ICI-ILD treatment, and patient management is currently guided by both clinical experience and trial guidelines. Both the Society of Immunotherapy of Cancer (SITC) and the ASCO have provided a definition on how to establish the severity grades [26]. Recently, the ASCO developed clinical practice guidelines based on expert consensus. The grading of pneumonitis was based on Common Terminology Criteria for Adverse Events definitions. Grade 1 was defined by an asymptomatic aspect, grade 2 by a symptomatic aspect as a limiting instrumental activities of daily living (ADL) and indicated medical intervention, grade 3 by severe symptoms limiting self-care ADL and indicated oxygen, and grade 4 by life-threatening respiratory compromise and indicated urgent intervention [25]. Moreover, according to the ESMO, the severity grade of pneumonitis was defined as 


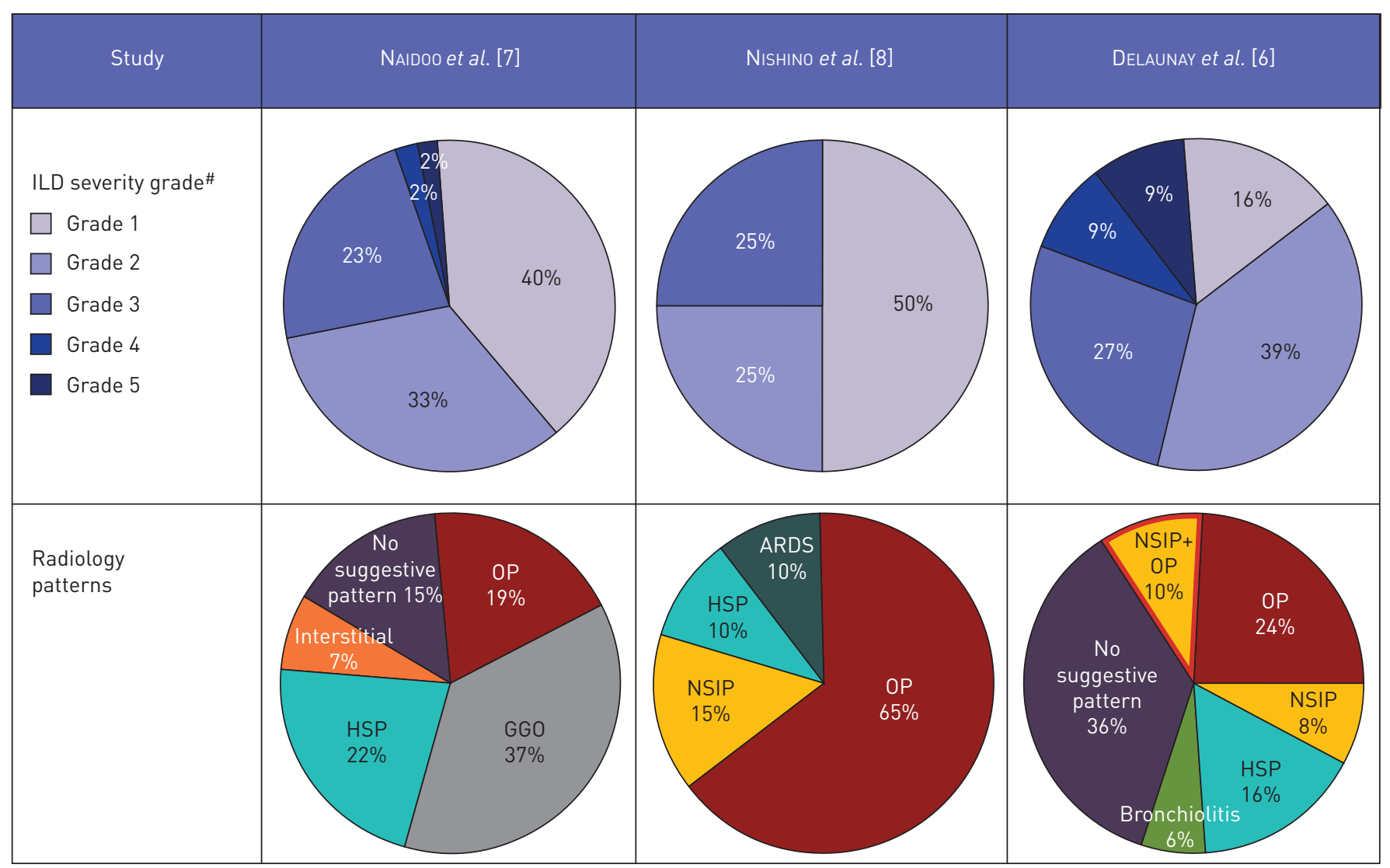

FIGURE 2 Comparison between three studies: severity grade and radiological patterns. OP: organising pneumoniae; HSP: hypersensitivity pneumonitis; NSIP: non-specific interstitial pneumoniae; GGO: ground-glass opacities; ARDS: acute respiratory distress syndrome. ${ }^{\#}$ : Common Terminology Criteria for Adverse Events 4:0.

follows: grade 1 radiographic changes only; grade $2 \mathrm{mid} /$ moderate new symptom occurrence (dyspnoea, cough and chest pain); and grade 3 and 4 severe new symptoms (new or worsening hypoxia, threat to life and breathing difficulties) [24].

Nowadays, ICI-ILD treatment is based on systemic steroids, as suggested in clinical trials, and is based on drug-related interstitial pneumonitis treatment [27]. For asymptomatic grade 1 we suggest either a close follow-up or low-dose steroids $\left(0.5-1 \mathrm{mg} \cdot \mathrm{kg}^{-1}\right)$. For grade 2 , steroids may systematically be proposed at $1-$ $2 \mathrm{mg} \cdot \mathrm{kg}^{-1}$ per day as recommended by the 2018 ASCO Guidelines [25]. Immunotherapy may be reconsidered if the toxic event recovers without complications. For grade $\geqslant 3$, a higher dose of steroids (2$4 \mathrm{mg} \cdot \mathrm{kg}^{-1}$ ) is recommended with drug holding. An intravenous steroid bolus may be considered for the most severe cases. Regarding any grade, CT monitoring should be proposed (figure 4). Steroid tapering should be conducted very slowly and carefully over $\geqslant 6$ weeks, as relapses of pneumonitis were reported during this period. It seems necessary to consider a longer period of corticosteroid taper ( $>6$ weeks) in order to avoid relapse. Moreover, according to the literature, it seems that if the pneumonitis episode is more severe (higher than grade 3 ) relapse occurs prematurely and mostly in cases where the treatment length is shorter $(<5$ weeks) $[28,29]$.

In cases of organised pneumonia, the treatment could be similar to the cryptogenic form of the disease [30]. Some examples of improvement after treatment are shown in figure 5.

Immunosuppressive drugs, such as infliximab or cyclophosphamide, have been proposed in clinical trials and initially approved by the US FDA for patients receiving ipilimumab, and especially for digestive toxicities such as colitis. A recent case report suggests that an interleukin-17 blockade may provide relief from immune-mediated skin and gastrointestinal toxic effects [31]. However, no data are currently available for either immunosuppressive drugs (especially infliximab or cyclophosphamide) or specific inhibition of interleukin-17 for ICI-ILD treatment.

In the series by NAIDOO et al. [19], immunosuppressive drugs were administered to five patients when high-dose steroids were insufficient; however, the use of an immunosuppressive drug may trigger a risk of 


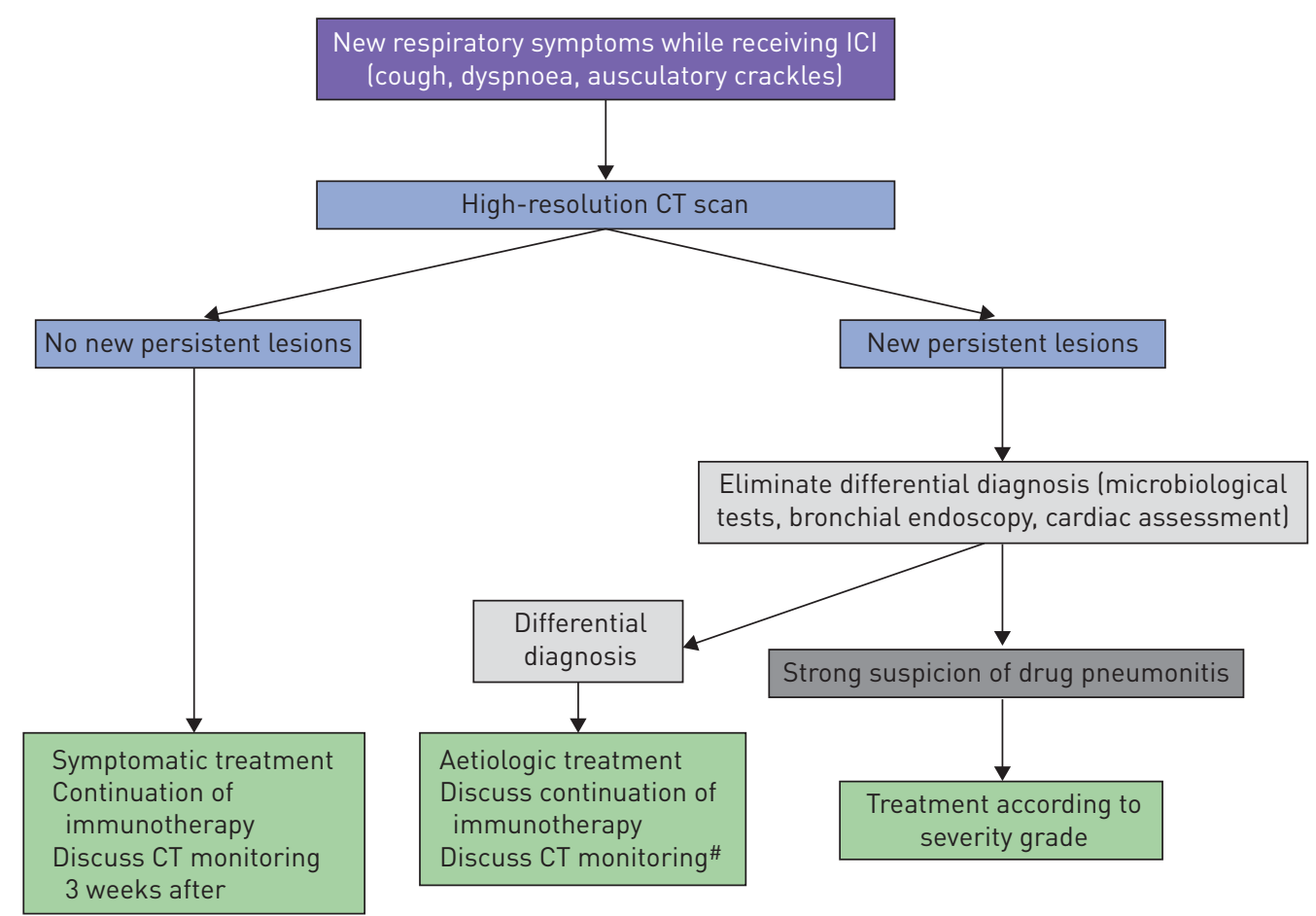

FIGURE 3 Suggested "decision tree" for interstitial lung disease induced by immune-checkpoint inhibitors (ICI) treatment. CT: computed tomography. " : progressive cancer lesions, infection, pulmonary embolism or heart failure.

infection. In their series, three out of five patients treated with anti-tumour necrosis factor (TNF), died as a consequence of sepsis: one from Pseudomonas aeruginosa, one from herpes simplex virus infection, and another one from mucormycosis [19]. SITC, ASCO and ESMO also suggest considering infliximab $\left(5 \mathrm{mg} \cdot \mathrm{kg}^{-1}\right)$, mycophenolate mofetil intravenously ( $1 \mathrm{~g}$ twice a day) or cyclophosphamide if there is no clinical improvement after steroids and supportive care [24-26]; however, the risk of infection and lung

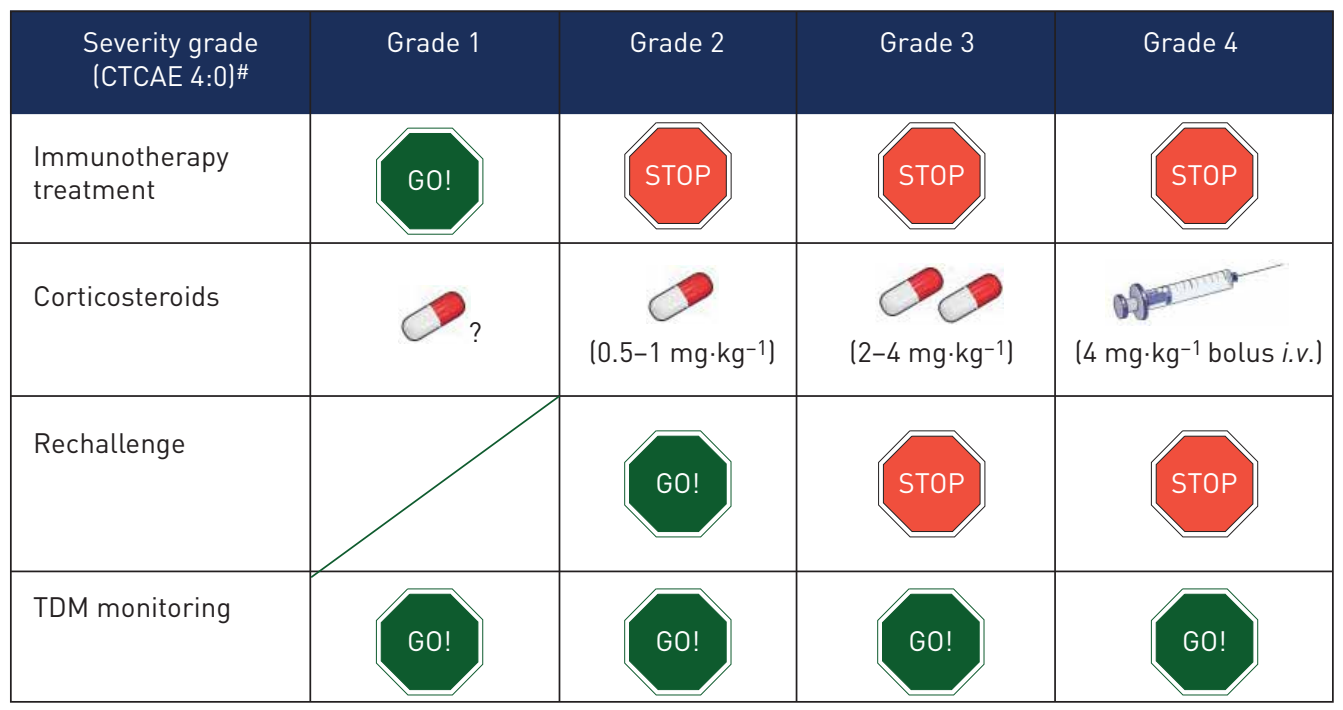

FIGURE 4 Suggested management according to severity grade. Grade 1: asymptomatic, clinical or diagnostic observation only; intervention not indicated; Grade 2: symptomatic, medical intervention indicated; limiting instrumental activities of daily living; Grade 3: severe symptoms; limiting self-care activities of daily living, oxygen indicated; Grade 4: life-threatening respiratory compromise; urgent intervention indicated le.g. tracheotomy or intubation). " : a disorder characterised by inflammation focally or diffusely affecting the lung parenchyma. 


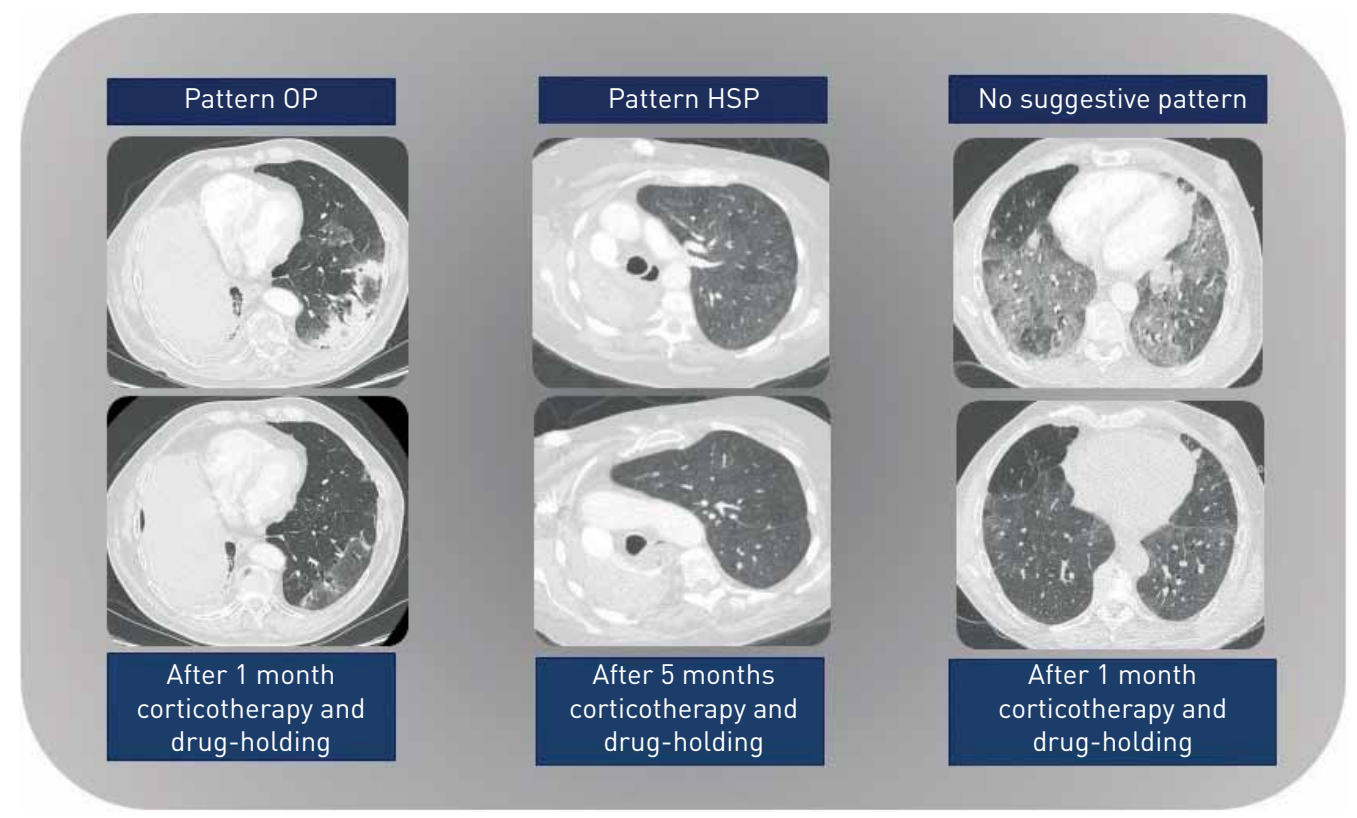

FIGURE 5 Interstitial lung disease induced by immune-checkpoint inhibitors improvement after treatment. $\mathrm{OP}$ : organising pneumoniae; HSP: hypersensitivity pneumonitis.

toxicity caused by anti-TNF drugs and the lack of knowledge on pathophysiological mechanisms of ICI-ILD make the use of this biotherapy particularly complex. This use should be discussed within a multidisciplinary board. Moreover, prophylactic antibiotherapy may be considered after microbiological assessments. If immunosuppression is prolonged, Pneumocystis prophylaxis also needs to be considered.

\section{Can we re-challenge patients with immunotherapy following lung toxicity?}

NAIDOo et al. [19] reported on 12 patients who were re-challenged. Overall, nine patients experienced no second event of pneumonitis while being re-challenged (eight were initially grade 1 and one was grade 2), and three experienced recurrent pneumonitis (one grade 1 and two grade 2).

We also reported on 10 patients who restarted immunotherapy. All re-challenged cases occurred after clinical resolution of pneumonitis. Among our 10 patients, recurrent ILD was observed in three cases. The second episode was successfully assessed by suspended ICI treatment plus steroids [6]. A recent retrospective study aimed to show the safety and benefit of retreatment following immune-related adverse events. When 68 out of 482 patients with NSCLC treated with anti-PD-L1 developed an immune-related adverse events they required treatment discontinuation; among these patients, 38 were retreated. Recurrent immune-related adverse events were more frequent when the initial immune-related adverse events required hospitalisation [32]. There is no established recommendation for a re-challenge, and options should be discussed by a multidisciplinary board, taking into account the expected benefits and risks for each patient.

\section{Other pulmonary toxicities}

Sarcoid-like granulomas induced by ipilimumab, nivolumab and pembrolizumab have been reported and are probably underestimated [33,34]. The radiological patterns are variable: from multiple pulmonary micronodular opacities or ground-glass infiltrates in subpleural and perihilar parenchyma to lymph node enlargement. Histologic examination could demonstrate non-necrotising granulomas [35]. This immune reaction may induce granulomas with epithelioid and giant cells surrounded by a ring of $\mathrm{CD} 4^{+}$and $\mathrm{CD} 8^{+}$ T-lymphocytes. The bronchoalveolar lavage typically demonstrates lymphocytic alveolitis with increased CD4:CD8 ratios. Organising pneumonia is characterised by lymphocytic interstitial inflammation [33]. Both of these entities occur from a T-helper-1 uncontrolled immune response but usually do not coexist. These results suggest, once again, an immune-mediated mechanism of toxicity.

Two cases of diaphragm myositis were reported with anti-PD-1 monotherapy and with a combination of anti-CTLA-4 and anti-PD-1 therapy. In the first case, anti-PD-1 treatment induced fatal respiratory failure with necrotising myositis of the diaphragm [36]. In the second case, this combination treatment induced fatal progressive hypoventilation [37]. 
Notably, two cases of tuberculosis have been reported in patients who were treated with anti-PD-1 agent for lymphoma and NSCLC [38]. A very recent study reported tuberculosis reactivation in patients treated with anti-PD-1 and suggested an immune reactivation syndrome [39].

Finally, one case of recurrent allergic bronchopulmonary aspergillosis has been reported [40]. The authors described that elevated serum immunoglobulin (Ig)E (up to $2514 \mathrm{kU} \cdot \mathrm{L}^{-1}$ ), and positive Aspergillus fumigatus IgE antibodies were consistent with a diagnosis of allergic bronchopulmonary aspergillosis. The patient was treated with both itraconazole and steroids that rapidly improved his condition.

\section{What is the impact of immune-related adverse events on patient outcomes?}

Whether immune-related adverse events are associated with clinical outcome remains unclear. Retrospective studies have found higher rates of disease control in patients who experienced immune-related adverse events [41]. A study assessed the benefit of atezolizumab (an anti-PD-L1 treatment) in patients with and without immune-related adverse events in the primary efficacy population $(\mathrm{n}=850)$. Overall survival favoured patients who received atezolizumab and presented immune-related adverse events versus those who did not (HR 0.79, 95\% CI 0.60-1.05) [42]. A recent prospective study included 38 patients with advanced NSCLC treated with anti-PD-1. In this study, patients with immune-related adverse events presented a higher objective response rate $(63.6 \%$ versus $7.4 \% \mathrm{p}<0.01)$ and longer progression free-survival (HR 0.10, 95\% CI 0.04-1.46; $\mathrm{p}=0.13$ ) [43].

These results should be confirmed because the correlation between toxicity and longer survival may be due to a lead-time bias. In other words, patients whose tumours progress show a higher mortality rate, whereas those who respond to immunotherapies have a longer treatment duration and are more likely to develop immune-related adverse events [44].

\section{Organisation}

Patients should be informed that most immune-related adverse events are reversible if they are detected early and specifically addressed. However, patients need to beware of the warning signs for the major organ toxicities linked to ICIs. Educational therapeutic programmes can optimise toxicity management. We also propose that each centre sets up a specialists' network that can manage the most frequent side-effects. Such a collaborative initiative could standardise and facilitate immune-related adverse event management, and this has already been reported in some centres, such as the Gustave Roussy Cancer Center (Villejuif, France) [45]. Moreover, local, regional or national dedicated boards specialised in immune-related adverse events are also very useful at collecting data and may guide patients towards the best strategies. Large registries and international efforts are also essential to avoid less frequent side-effects.

\section{Conclusion}

Lung toxicities associated with ICI treatment are not exceptional. They usually occur during the first few months and are linked to nonspecific clinical symptoms but have suggestive radiological signs. Awareness of radiological patterns and clinical manifestations of ICI-ILD are necessary to rapidly diagnose and treat these possible serious events. Endoscopy, including bronchoalveolar lavage and transbronchial lung biopsies can refine a diagnosis by excluding pulmonary infection and showing lymphocytic alveolitis.

Additional studies are required to further understand the clinical characteristics and chronology of these adverse effects and to clarify the mechanism(s) leading immunotherapy to cause these immune-related events.

Conflict of interest: M. Delaunay has nothing to disclose. G. Prévot has nothing to disclose. S. Collot has nothing to disclose. L. Guilleminault has nothing to disclose. A. Didier has nothing to disclose. J. Mazières reports grants and personal fees from AstraZeneca, BMS and Roche, and personal fees from Novartis, MSD, Pfizer and Pharmamar, outside the submitted work.

\section{References}

1 Rosenberg JE, Hoffman-Censits J, Powles T, et al. Atezolizumab in patients with locally advanced and metastatic urothelial carcinoma who have progressed following treatment with platinum-based chemotherapy: a single-arm, multicentre, phase II trial. Lancet 2016; 387: 1909-1920.

2 Ferris RL, Blumenschein G, Jr., Fayette J, et al. Nivolumab for recurrent squamous-cell carcinoma of the head and neck. N Engl J Med 2016; 375: 1856-1867.

3 Ansell SM, Lesokhin AM, Borrello I, et al. PD-1 blockade with nivolumab in relapsed or refractory Hodgkin's lymphoma. N Engl J Med 2015; 372: 311-319.

4 Michot JM, Bigenwald C, Champiat S, et al. Immune-related adverse events with immune checkpoint blockade: a comprehensive review. Eur J Cancer 2016; 54: 139-148.

5 Nishino M, Giobbie-Hurder A, Hatabu $\mathrm{H}$, et al. Incidence of programmed cell death 1 inhibitor-related pneumonitis in patients with advanced cancer: a systematic review and meta-analysis. JAMA Oncol 2016; 2: $1607-1616$. 
6 Delaunay M, Cadranel J, Lusque A, et al. Immune-checkpoint inhibitors associated with interstitial lung disease in cancer patients. Eur Respir J 2017; 50: 1700050.

7 Naidoo J, Wang X, Woo KM, et al. Pneumonitis in patients treated with anti-programmed death-1/programmed death ligand 1 therapy. J Clin Oncol 2017; 35: 709-717.

8 Nishino M, Ramaiya NH, Awad MM, et al. PD-1 inhibitor-related pneumonitis in advanced cancer patients: radiographic patterns and clinical course. Clin Cancer Res 2016; 22: 6051-6060.

9 Langer CJ, Gadgeel SM, Borghaei H, et al. Carboplatin and pemetrexed with or without pembrolizumab for advanced, non-squamous non-small-cell lung cancer: a randomised, phase 2 cohort of the open-label KEYNOTE-021 study. Lancet Oncol 2016; 17: 1497-1508.

10 Antonia SJ, Villegas A, Daniel D, et al. Durvalumab after chemoradiotherapy in stage III non-small-cell lung cancer. N Engl J Med 2017; 377: 1919-1929.

11 Toh CK, Wong EH, Lim WT, et al. The impact of smoking status on the behavior and survival outcome of patients with advanced non-small cell lung cancer: a retrospective analysis. Chest 2004; 126: 1750-1756.

12 Fujimoto D, Morimoto T, Ito J, et al. A pilot trial of nivolumab treatment for advanced non-small cell lung cancer patients with mild idiopathic interstitial pneumonia. Lung Cancer 2017; 111: 1-5.

13 Chiang CL, Chen YW, Wu MH, et al. Radiation recall pneumonitis induced by epidermal growth factor receptortyrosine kinase inhibitor in patients with advanced nonsmall-cell lung cancer. J Chin Med Assoc 2016; 79: 248-255.

14 Khunger M, Rakshit S, Pasupuleti V, et al. Incidence of pneumonitis with use of programmed death 1 and programmed death-ligand 1 inhibitors in non-small cell lung cancer: a systematic review and meta-analysis of trials. Chest 2017; 152: 271-281.

15 Tabchi S, Messier C, Blais N. Immune-mediated respiratory adverse events of checkpoint inhibitors. Curr Opin Oncol 2016; 28: 269-277.

16 Johnson DB, Sullivan RJ, Ott PA, et al. Ipilimumab therapy in patients with advanced melanoma and preexisting autoimmune disorders. JAMA Oncol 2016; 2: 234-240.

17 Menzies AM, Johnson DB, Ramanujam S, et al. Anti-PD-1 therapy in patients with advanced melanoma and preexisting autoimmune disorders or major toxicity with ipilimumab. Ann Oncol 2017; 28: 368-376.

18 Leonardi GC, Gainor JF, Altan M, et al. Safety of programmed death-1 pathway inhibitors among patients with non-small-cell lung cancer and preexisting autoimmune disorders. J Clin Oncol 2018; 36: 1905-1912.

19 Naidoo J, Schindler K, Querfeld C, et al. Autoimmune bullous skin disorders with immune checkpoint inhibitors targeting PD-1 and PD-L1. Cancer Immunol Res 2016; 4: 383-389.

20 Travis WD, Costabel U, Hansell DM, et al. An official American Thoracic Society/European Respiratory Society statement: update of the international multidisciplinary classification of the idiopathic interstitial pneumonias. Am J Respir Crit Care Med 2013; 188: 733-748.

21 Barjaktarevic IZ, Qadir N, Suri A, et al. Organizing pneumonia as a side effect of ipilimumab treatment of melanoma. Chest 2013; 143: 858-861.

22 Travis WD, Brambilla E, Noguchi M, et al. International Association for the Study of Lung Cancer/American Thoracic Society/European Respiratory Society International multidisciplinary classification of lung adenocarcinoma. J Thorac Oncol 2011; 6: 244-285.

23 Satake N, Nagai S, Kawatani A, et al. Density of phenotypic markers on BAL T-lymphocytes in hypersensitivity pneumonitis, pulmonary sarcoidosis and bronchiolitis obliterans with organizing pneumonia. Eur Respir J 1993; 6 : $477-482$.

24 Haanen J, Carbonnel F, Robert C, et al. Management of toxicities from immunotherapy: ESMO Clinical Practice Guidelines for diagnosis, treatment and follow-up. Ann Oncol 2017; 28: Suppl. 4, iv119-iv142.

25 Brahmer JR, Lacchetti C, Schneider BJ, et al. Management of immune-related adverse events in patients treated with immune checkpoint inhibitor therapy: American Society of Clinical Oncology clinical practice guideline. J Clin Oncol 2018; 36: 1714-1768.

26 Puzanov I, Diab A, Abdallah K, et al. Managing toxicities associated with immune checkpoint inhibitors: consensus recommendations from the Society for Immunotherapy of Cancer (SITC) toxicity management working group. J Immunother Cancer 2017; 5: 95.

27 Bonniaud P, Georges M, Favrolt N, et al. [Drug-induced interstitial lung diseases]. Rev Prat 2014; 64: 951-956.

28 Asher N, Marom EM, Ben-Betzalel G, et al. Recurrent pneumonitis in patients with melanoma treated with immune checkpoint inhibitors. Oncologist 2019; 24: 640-647.

29 de Jong C, Peters BJM, Schramel F. Recurrent episodes of nivolumab-induced pneumonitis after nivolumab discontinuation and the time course of carcinoembryonic antigen levels: a case of a 58-year-old woman with non-small cell lung cancer. Chemotherapy 2018; 63: 272-277.

30 Cordier JF. Cryptogenic organising pneumonia. Eur Respir J 2006; 28: 422-446.

31 Esfahani K, Miller WH, Jr. Reversal of autoimmune toxicity and loss of tumor response by interleukin-17 blockade. N Engl J Med 2017; 376: 1989-1991.

32 Santini FC, Rizvi H, Plodkowski AJ, et al. Safety and efficacy of re-treating with immunotherapy after immune-related adverse events in patients with NSCLC. Cancer Immunol Res 2018; 6: 1093-1099.

33 Berthod G, Lazor R, Letovanec I, et al. Pulmonary sarcoid-like granulomatosis induced by ipilimumab. J Clin Oncol 2012; 30: e156-e159.

34 Faviez G, Bousquet E, Rabeau A, et al. [Sarcoid-like granulomatosis in cancer patients treated with immune checkpoints inhibitors]. Rev Mal Respir 2018; 35: 963-967.

35 Nishino M, Sholl LM, Awad MM, et al. Sarcoid-like granulomatosis of the lung related to immune-checkpoint inhibitors: distinct clinical and imaging features of a unique immune-related adverse event. Cancer Immunol Res 2018; 6: 630-635.

36 Haddox CL, Shenoy N, Shah KK, et al. Pembrolizumab-induced bulbar myopathy and respiratory failure with necrotizing myositis of the diaphragm. Ann Oncol 2017; 28: 673-675.

37 John S, Antonia SJ, Rose TA, et al. Progressive hypoventilation due to mixed CD8(+) and CD4(+) lymphocytic polymyositis following tremelimumab-durvalumab treatment. J Immunother Cancer 2017; 5: 54.

38 Reungwetwattana T, Adjei AA. Anti-PD-1 antibody treatment and the development of acute pulmonary tuberculosis. J Thorac Oncol 2016; 11: 2048-2050. 
39 Picchi H, Mateus C, Chouaid C, et al. Infectious complications associated with the use of immune checkpoint inhibitors in oncology: reactivation of tuberculosis after anti PD-1 treatment. Clin Microbiol Infect 2018; 24: 216-218.

40 Pradere P, Michot JM, Champiat S, et al. Allergic broncho-pulmonary aspergillosis following treatment with an anti-programmed cell death protein 1 monoclonal antibody therapy. Eur J Cancer 2017; 75: 308-309.

41 Osorio JC, Ni A, Chaft JE, et al. Antibody-mediated thyroid dysfunction during T-cell checkpoint blockade in patients with non-small-cell lung cancer. Ann Oncol 2017; 28: 583-589.

42 von Pawel J, Syrigos K, Mazieres J, et al. 1314PAssociation between immune-related adverse events (irAEs) and atezolizumab efficacy in advanced NSCLC: analyses from the phase III study OAK. Ann Oncol 2017; 28: Suppl. 5, $\mathrm{mdx} 380.017$.

43 Sato K, Akamatsu H, Murakami E, et al. Correlation between immune-related adverse events and efficacy in non-small cell lung cancer treated with nivolumab. Lung Cancer 2018; 115: 71-74.

44 June $\mathrm{CH}$, Warshauer JT, Bluestone JA. Is autoimmunity the Achilles' heel of cancer immunotherapy? Nat Med 2017; 23: 540-547.

45 Champiat S, Lambotte O, Barreau E, et al. Management of immune checkpoint blockade dysimmune toxicities: a collaborative position paper. Ann Oncol 2016; 27: 559-574. 